\title{
Perinatal complications associated with neuraxial blocks
}

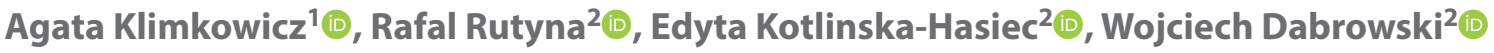 \\ ${ }^{1}$ Department of Anaesthesiology and Intensive Care, Independent Public Teaching Hospital No 4 in Lublin, Poland \\ 2 Clinic of Anaesthesiology and Intensive Therapy, Medical University of Lublin, Poland
}

\begin{abstract}
Regional techniques are the gold standard of obstetric anaesthesia. In both vaginal and Caesarean section deliveries, neuraxial blocks are the most frequently used methods for relieving pain. Although it provides excellent analgesia, regional anaesthesia is associated with certain adverse side effects and possible complications. In this narrative review, we bring together all available data and create a catalogue of complications resulting from the use of perinatal neuraxial anaesthesia which we divide according to their severity and the duration of their impact on patients' health. We focus on complications that have significant or long-term consequences. Even though their incidence is low at 1:1600 neuraxial anaesthetics performed, we believe that better understanding of the possible severe problems that can result from regional anaesthesia procedures would enhance the overall safety of patients during labour, delivery, and the postpartum period. Despite the pivotal role neuraxial techniques play in providing anaesthesia for parturients, there is a lack of good quality studies on the incidence of complications. We believe that a thorough assessment of the occurrence of complications should be carried out by analysing data from nationwide medical databases. By analysing the adverse side effects, both qualitatively and quantitatively, we think it possible to further improve the quality of patient care.
\end{abstract}

Key words: complications; adverse effects; neuraxial anaesthesia; obstetrical anaesthesia; epidural analgesia; spinal anaesthesia

Ginekologia Polska 2021; 92, 6: 446-452

\section{INTRODUCTION}

Childbirth is one of the most painful events in the lives of the vast majority of women. Among the many currently available pain relief methods, neuraxial techniques - including subarachnoid blocks, epidural blocks, and combined spinal-epidural (CSE) blocks - are considered the gold standards [1]. The difference between the anatomical placement of an epidural and spinal block is showed in Figure 1. Although these techniques are well-known and have been commonly used in anaesthesia since the $19^{\text {th }}$ century, their prevalence in obstetrics varies widely according to organisational and financial factors, local medical policies, and sometimes even the individual preferences of the anaesthesiologist. Generally, epidurals are the most prevalent form of anaesthesia during vaginal delivery. One of the biggest advantages of this method is the possibility of utilizing the epidural catheter that is already in place to induce anaesthesia in the event of an emergency Caesarean section. In cases of Caesarean sections performed without an attempt of a vaginal delivery, spinal blocks are most commonly used. There is a lack of good quality data regarding how often neuraxial anaesthesia is used for pain relief during childbirth. In 2019 it is estimated that around 140 million births took place worldwide. The literature shows extreme variation between different countries in the use of neuraxial labour analgesia, ranging from $0 \%$ to over $80 \%$ [2]. This variation results not only from a country income but also from cultural and social factors. In Poland, unfortunately, there are no up-to-date scientific data on the prevalence of different methods of alleviating labour pain.

The provision of high-quality services requires in-depth knowledge of anaesthetic techniques and systematic oversight of the number of complications related to neuraxial techniques. An analysis carried out by the American Society for Obstetric Anaesthesia and Perinatology found that serious adverse events occur in one out of every 1636 central blocks among obstetrics patients [3]. It is difficult, however, to determine unequivocally whether a given complication is

\author{
Corresponding author: \\ Agata Klimkowicz \\ Department of Anaesthesiology and Intensive Care, Independent Public Teaching Hospital № 4 in Lublin, Jaczewskiego 8, 20-090 Lublin, Poland \\ e-mail: agata.klimkowicz@gmail.com
}

This article is available in open access under Creative Common Attribution-Non-Commercial-No Derivatives 4.0 International (CC BY-NC-ND 4.0) license, allowing to download articles and share them with others as long as they credit the authors and the publisher, but without permission to change them in any way or use them commercially. 


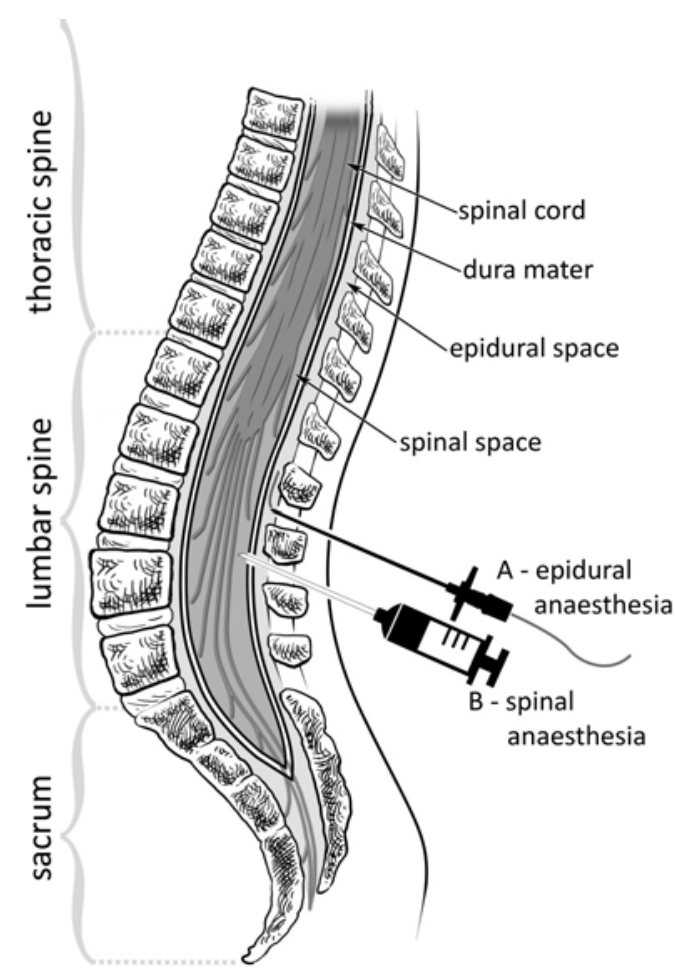

Figure 1. Anatomical placement of neuraxial blocks; $A$ - epidural anaesthesia; B - spinal anaesthesia

causally linked to the regional technique used. Nevertheless, this does not explain the lack or marginalisation of analysis of the incidence and causes of adverse events resulting from obstetric anaesthesia procedures. This following narrative review aims to qualitatively and quantitatively describe the undesirable effects and complications of central blocks in patients in the perinatal period [4].

The literature does not precisely define the term "complication". The inconsistent use of the various terms - complications, adverse effects, side effects, sequelae - significantly impedes efforts to precisely define "complications". Semantically, these terms differ from each other, yet they are not uniformly or consistently employed in scientific literature. The term "side effect" deserves to be explained more clearly, as it refers to an effect secondary to that which is initially intended from the given medication or procedure. Therefore, side effects are anticipated additional consequences that may be either positive or negative for the patient's health [1]. For the purpose of our review, we have considered any deterioration of a patient's health for which a cause-and-effect relationship to the performance of a regional block can be demonstrated as a complication.

We searched MEDLINE, Google Scholar and Scopus using queries based on keywords such as "obstetric anaesthesia", "labour anaesthesia", "neuraxial anaesthesia", "adverse effects", and "complications". Initially, we reviewed the abstracts of 854 scholarly articles. Then, the papers were analysed for complications related to anaesthesia in obstetrics. After a thorough analysis, we considered eight articles to be especially applicable to our review [3,5-11].

Due to the large number of possible complications, we organised them according to the degree and duration of their impact on a woman's health. The highly subjective nature of this division should be emphasised, as it is not always valid for every individual case. Short-lived complications with a low potential for harm are presented in Table 1. Among the many complications falling into the category of "side effects", the most common is hypotension, which is an expected side effect of a neuraxial block. It is caused by a blockade of sympathetic neurons and will occur in varying degrees of severity in most patients. In extreme cases, it may affect organ perfusion in the mother or may impair maternal-fetal blood flow. In most cases, hypotension is transient and easily treatable and does not cause any long-term consequences.

\section{FEVER}

A meta-analysis published in 2018 in the Cochrane Database of Systematic Reviews showed that the use of epidural anaesthesia doubles the risk of temperatures above $38^{\circ} \mathrm{C}$ compared to women who received opioids to relieve labour pain [12]. The incidence of this complication varies widely, between $1 \%$ and $33 \%$, from study to study and is most commonly observed among primiparas [15]. Randomized studies contradict the previously observed correlation between the frequency of fever and prolonged epidural catheterization. However, the aetiology of the association of elevated body temperature with neuraxial blocks is unclear. Possible causes include the systemic inflammatory reaction induced by the neuraxial block, as well as reduced heat loss since anaesthesia reduces the perspiration and hyperventilation that would otherwise be caused by pain [1]. However, it should not be forgotten that fever in patients during labour may be indicative of an intra-amniotic infection. In addition, some side effects of neuraxial anaesthesia, such as motor block, may prolong labour and thus contribute indirectly to the risk of developing chorioamnionitis. Due to the lack of tests that can clearly differentiate between fever resulting from a neuraxial block and fever that is symptomatic of a serious

Table 1. Complications with low impact on women's health

\begin{tabular}{|l|l|}
\hline Hypotension - spinal anaesthesia & $25-71.25 \%[3]$ \\
\hline Hypotension - epidural anaesthesia & $8-30.7 \%[12]$ \\
\hline Pruritus & $1.3-85 \%[13]$ \\
\hline Nausea and vomiting & $3.2-34 \%[13]$ \\
\hline Urinary retention & $0.006-3.4 \%[10]$ \\
\hline Shivering & $36-71 \%[14]$ \\
\hline
\end{tabular}


infection, it is necessary to remain vigilant and - after performing a physical examination and excluding other sources of fever - consider implementing antibiotic therapy.

\section{INSUFFICIENT PAIN RELIEF OR FAILED NEURAXIAL BLOCK}

A neuraxial block is considered ineffective if it fails to provide satisfactory pain reduction or anaesthesia, or when the anaesthesia is insufficient to perform the surgery and an alternative method is necessary. Studies analysing the causes of neuraxial anaesthesia failure implicate emergency Caesarean sections and high BMI values as well as the obstetric histories of patients and the choices of anaesthesia techniques as possible causes [16]. With epidural and CSE blocks used for analgesia during labour and vaginal delivery, the failure rate ranges from $12 \%$ to $23 \%[16,17]$. In these cases, considering the individual case of each patient, we may offer the parturient a reinsertion of the epidural catheter or another pain relief method. For Caesarean sections, insufficient anaesthesia is usually the result of converting a labour analgesia epidural into a surgical anaesthetic block using the same catheter that had been previously sited in the labour suite. This occurs in $7 \%$ to $23 \%$ of cases, up to half of which may require conversion to general anaesthesia according to some studies [16]. Spinal anaesthesia is characterised by a considerably lower incidence of inadequate blockade (2.7-5\%) and the need for general anaesthesia (1.2-2\%) [7].

The use of ultrasonography for placing neuraxial blocks seems to be of significant benefit. Clinical observations show significantly lower risks of incomplete blocks and failures of epidural anaesthesia in patients for whom ultrasound visualisation was used during neuraxial anaesthesia placement [18]. This technique is particularly helpful in patients with anatomical abnormalities.

\section{MOTOR BLOCK}

There has been a lack of good quality research on the incidence of motor blocks. Neuraxial techniques used for vaginal delivery aim to switch off the conduction of pain impulses in the nervous pathways without simultaneously impairing motor function. This is often referred to as a "walking epidural", as it allows the patient to move freely. Excessive, undesirable motor block may inhibit activity in the first stage, as well as hinder and lengthen the second stage of labour. As a result, it may increase the number of deliveries that require the use of forceps or a vacuum [1]. The incidence of motor block is largely correlated to the anaesthesiologist's experience in obstetric anaesthesia. Although there is some individual variability in reactions to drugs, in the majority of cases, we can avoid motor block and other complications, such as surgical delivery, through the use of low concentrations of local anaesthetics in combination with opioid drugs [12].

We can use the patient-controlled epidural anaesthesia (PCEA) system, which enables the patient to administer pre-programmed drug doses using an infusion pump, in hopes of better controlling the level of anaesthesia. However, the literature does not conclusively establish the superiority of this method over physician-administered boluses of local anaesthetic.

\section{POST DURAL PUNCTURE HEADACHE}

Post dural puncture headache is defined as pain occurring within five days after the lumbar puncture and resulting from cerebrospinal fluid leakage. According to the International Classification of Headache Disorders (ICHD-3), it is commonly accompanied by symptoms such as neck stiffness and subjective hearing loss [19]. In most cases, symptoms appear during the first 72 hours after the procedure, but in rare cases, they can develop up to 14 days later. In differential diagnosis, we must consider pre-eclampsia or eclampsia, migraine, meningitis, and even CNS bleeding or venous sinus thrombosis. According to the diagnostic criteria provided in the ICHD-3, patients should be diagnosed with post dural puncture headache only after competing diagnoses have been ruled out. One prospective cohort study showed that up to $40 \%$ of women experience headache during the postpartum period, with less than five percent of cases being post dural puncture headache [20]. The incidence of this complication is around one in 114 neuraxial blocks, but it varies greatly depending on the type of neuraxial technique [3]. In particular, it is often associated with epidural anaesthesia during which an unintentional dural puncture (UDP) may occur, increasing the risk of post dural puncture headache to over $50 \%$ [21]. Post dural puncture headache results significantly less frequently from spinal blocks. The management of this condition depends on the severity of the symptoms. In some cases, fluid therapy, administration of nonsteroidal anti-inflammatory drugs, caffeine, and bed rest are sufficient. In cases where this conservative management fails, the treatment of choice is an autologous blood patch - a procedure in which a low volume of patients own blood is injected into their epidural space to create a seal to stop a leak of cerebrospinal fluid — which is about $80 \%$ effective [3].

\section{HIGH BLOCK}

The high block is one of the most serious complications in obstetric anaesthesiology. It occurs in one out of every 4,336 neuraxial blocks performed when the area of the sympathetic, sensory, and motor block reaches the level of the cervical segments of the spinal cord [3]. Its symptoms depend on the extent of the blocked area of the nervous sys- 
tem and include nausea, dizziness, and respiratory distress up to loss of consciousness or cardiac arrest [10]. The patient may sometimes need to be intubated and mechanically ventilated. If there are no further consequences of respiratory or circulatory failure, the patient will most likely recover once the effects of local anaesthetics have subsided. The actual mechanism of this complication is not well-understood, but the anaesthesiologist's choice of technique or the type of the anaesthetic drug used may play a role [3].

For epidural anaesthesia, it is assumed that the dose of the drug, which may be as high as $20 \mathrm{~mL}$, will be administered to the subarachnoid space instead of to the epidural space. High block may occur during both the one bolus technique and the continuous technique using a catheter in the epidural space to administer successive doses of local anaesthetics. Because an epidural catheter may spontaneously move to the subarachnoid space at any given time after it is placed, utmost vigilance is essential during the administration of subsequent doses of drugs [3].

\section{RESPIRATORY DEPRESSION}

Respiratory depression due to neuraxial block is assumed to be an extremely rare, extremely dangerous complication of neuraxial anaesthesia. The available data do not document the incidence of this complication in women in the perinatal period. The most frequently cited cause of respiratory depression is the administration of opioid drugs. Studies carried out on non-obstetric patients associated neuraxial anaesthesia with a $0.01-7 \%$ risk of respiratory depression [22]. The severity of the depressive effect depends on the dose, the route of administration, and the pharmacokinetics of the drug itself. The onset of symptoms can occur from a few minutes to several hours after the neuraxial block. Therefore, it is necessary to carefully assess the patient's status at each stage of anaesthesia and in the period immediately after its completion; The American Society of Anesthesiologists recommends monitoring the respiratory performance values for at least 24 hours after epidural or subarachnoid administration of morphine and for at least two hours after analogous administration of fentanyl or sufentanil [23]. Respiratory disorders may also occur as a result of excessive block and be related to the paralysis of the respiratory muscles or respiratory centre, as described above.

\section{SERIOUS NEUROLOGICAL DISORDERS}

Both pregnancy and childbirth, as well as anaesthesia itself, can be directly linked to the development of neurological dysfunctions. These most often manifest as sensory deficits in the lower extremities and buttocks or as deficits in motor function. This damage may occur in several forms, the most important of which include direct nerve damage; injury due to compression, which itself is caused by a haematoma or spinal canal abscess; and chemical damage caused by the administered drug. Although neuraxial anaesthesia procedures are an undeniable intervention in the patient's nervous system, they are not the most common cause of these symptoms. The risk of serious neurological injury resulting from neuraxial anaesthesia varies from 1:35,923 to 1:237,000 blocks performed [3]. Obstetric injuries, also called intrinsic maternal obstetric palsies, are more commonly caused by compression of the nerves or the blood vessels supplying them by the child passing through the birth canal; inappropriate position of the patient, especially in the second stage of labour; or direct injury during instrumental delivery [9]. The association of nerve damage with the performance of neuraxial blocks can only be confirmed by the occurrence of atypical neurological symptoms, such as pain and paraesthesia, during the procedure; these symptoms most often occur in the area of the neurological deficit observed after childbirth [5]. One of the most frequently described injuries is Cauda Equina Syndrome (CES), which consists of injury to the L5-S5 nerve roots and is associated with numbness of the skin in the perineal region, weakness of the lower limbs, and sphincter muscle dysfunction [5]. The damage is most often not permanent, and the symptoms tend to spontaneously resolve, but it may take several months to fully recover. Cases of permanent damage due to central blockade have been reported very infrequently (0.2-1.2:100000) [6].

\section{DEEP INFECTION}

Serious infection is a rare complication of neuraxial block, but its occurrence may threaten the patient's life. It manifests in the form of meningitis or spinal canal abscess and occurs in 1:62,866 to 1:145,000 neuraxial blocks performed [8]. Most often, meningitis is a complication of procedures during which the dura was punctured, whether intentionally or not, while spinal canal abscesses usually result from the use of epidurals. Research implicates Viridians streptococci as the pathogen responsible for almost $50 \%$ of iatrogenic cases of meningitis [24]. It is thought that the bacteria probably originate from the upper respiratory tract of the person performing the procedure, which highlights the importance of maintaining proper aseptic protocols. The risk of developing meningitis is also greater in patients with bacteriemia, which is more common in women who, among other things, have previously struggled with genitourinary infection or who required manual extraction of the placenta. In such cases, the pathogen causing meningitis may be B-group Streptococcus [25]. A diagnosis of meningitis should be considered in cases of the presentation of symptoms such as fever, severe headache with accompanying nausea and vomiting, neck stiffness, and 
other neurological symptoms. Although the most common symptom of spinal canal abscess is back pain, this is a frequent complaint among women in the postpartum period and is often directly related to pregnancy or labour and delivery itself. If, however, the back pain is accompanied by symptoms of neurological deficiency - particularly sphincter insufficiency - and fever, this classic triad of symptoms points instead to an infection within the central nervous system. The risk of abscess is greater in patients who receive prolonged maintenance of an epidural catheter or who have a history of diabetes or immunodeficiency [5]. Magnetic resonance imaging (MRI) with gadolinium contrast is the diagnostic tool of choice. In some cases, surgical intervention is required, which increases the chances of regaining lost motor functions. Both in the case of meningitis and of spinal canal abscess, it is necessary to monitor inflammation marker levels, perform appropriate culture tests, and implement adequate antimicrobial therapy.

\section{SPINAL EPIDURAL HEMATOMA}

A haemorrhage into the central nervous system is a rare but serious complication that occurs less frequently in obstetric patients, at a rate of 1:168,000-1:251,469, than in other populations [5]. This complication is significantly more common in women suffering from coagulation disorders or taking anticoagulant or antiplatelet medications. Nowadays, many patients take acetylsalicylic acid and low-molecular-weight heparins for the prevention of obstetric complications. Early withdrawal of treatment with these drugs is therefore recommended in patients who wish to receive epidural analgesia for labour and delivery or who are scheduled for a Caesarean section. In addition, pregnancy predisposes women to idiopathic thrombocytopenic purpura (ITP) or low platelet count during HELLP syndrome. Platelet counts below $80,000 / \mathrm{mm}^{3}$, regardless of the cause, are considered by most anaesthesiology specialists to be a contraindication for the use of neuraxial anaesthesia techniques [9]. The symptoms' progression usually depends on how rapidly the hematoma forms. The most common symptoms include sensory deprivation, motor dysfunction of the lower limbs, and sphincter function disorders. If a spinal haematoma is suspected, a spinal MRI is necessary, and a neurosurgical intervention may be required, in which case the surgery should be performed as soon as possible to minimise long-term neurological deficits.

\section{SUDDEN CARDIAC ARREST}

Sudden cardiac arrest in pregnant women is rare and carries a $60 \%$ survival rate [26]. According to studies conducted in the U.S., cardiac arrest occurred approximately once in every 12,000 hospitalizations related to childbirth [27]. In the Serious Complication Repository Project of the Society for Obstetric Anesthesia and Perinatology, of nearly 250,000 births, sudden cardiac arrest occurred in 43 cases, $88 \%$ of which were Caesarean section deliveries. The most common causes included haemorrhage, amniotic fluid embolism, and pre-existing cardiac conditions [3]. In the same study, the incidence of sudden cardiac arrest because of anaesthesia was estimated at 1:128,398 [3]. The results of the Royal College of Anaesthetists' $3^{\text {rd }}$ National Audit Project (NAP3), which did not describe a single case of cardiac arrest in a group of seven hundred thousand patients, suggest an extremely low risk of cardiac arrest due to the use of neuraxial anaesthesia in the perinatal period [6]. The U.K.'s Cardiac Arrest in Pregnancy Study (CAPS), analysing all cases of cardiac arrest among pregnant women in which basic life support measures were started, found that almost $25 \%$ of them were related to anaesthesia [26]. Of these 17 cases of sudden cardiac arrest linked with anaesthetic management, as many as 10 of them resulted from high block from neuraxial anaesthesia [26]. Despite significant differences in data on the incidence of sudden cardiac arrest related to anaesthesia, in all cases reported in the aforementioned studies, resuscitation was successful, and the patients survived.

\section{LOCAL ANAESTHETIC SYSTEMIC TOXICITY}

Local anaesthetic (LA) medication acts by blocking sodium channels and thus stopping the conduction of nerve impulses. Such an important interference in the human body may cause serious consequences, especially in the cardiovascular and nervous systems. There is a linear relationship between the concentration of LAs in the blood serum and the severity of symptoms. The initial symptoms are cardiac dysrhythmias and pathognomy changes in sensation within the mouth and tongue, with patients often reporting a metallic taste. As the blood serum concentration of LA increases, the patient may experience seizures, heart dysrhythmias that result in cardiac failure, and finally, loss of consciousness and cardiac arrest. Due to the potentially fatal consequences of local anaesthetic systemic toxicity (LAST), the guidelines for performing obstetric anaesthesia recommend equipping the labour and delivery ward not only with a cardiopulmonary resuscitation kit, but also a $20 \%$ lipid emulsion available for immediate administration. This emulsion is intended to bind to $L A$ and thus reduce its blood serum concentration [28]. If the patient experiences any symptoms that might be indicative of LAST, the LA infusion should be immediately discontinued, the resuscitation team should be called in, and $100 \%$ oxygen should be administered to the patient with instructions to breathe deeply.

In the perinatal period, the most common cause of toxicity symptoms is the unintentional injection of LA into a venous vessel in the epidural space. This complication is less common with spinal anaesthesia due to the much smaller 
dose of LA used. It is worth noting that, even if the technique is properly performed, we can never be certain that the catheter is properly positioned in the epidural space. It should also be stressed that even a catheter correctly inserted in the epidural space may be displaced during labour. To minimise the risk of intravascular or subarachnoid administration of the medication, a test dose - i.e., a small amount of LA given before the full intended dose - should always be used [1]. This complication is observed in 1:2,500 epidural anaesthetics performed in the general population; however, the incidence of this complication in the obstetric population has not been determined [28]. It seems that, in the obstetric population, LAST is not a common complication, as low-concentration solutions of LAs are administered, and the overall dose is not high.

\section{SUMMARY}

The widespread availability of pain relief in the perinatal period represents a significant milestone in the development of human civilisation. The improvement and increasingly widespread use of regional anaesthesia techniques in obstetric anaesthesiology has led to a reduction in perinatal mortality associated with anaesthesia. In order to maintain the quality and increase the safety of medical procedures, it is essential to understand the complications involved. While the catalogue of possible complications of regional anaesthesia techniques is well-known, the lack of good quality studies assessing their quantitative distribution is striking. This is also reflected in the very high dispersion of the incidence of specific complications in the analyses carried out by different authors. The frequency of adverse events may also be distorted due to the use of very small, unrepresentative samples in many studies. These problems may cause us to underestimate importance of some of complications and to overestimate the importance of others. On the other hand, some of the variability in the incidence of particular complications is related to local variations in the model of perinatal care and the resulting use of neuraxial blocks in obstetric patients. The literature also highlights the impact of the experience and skills of anaesthesiologists on the number of complications. This thesis can be applied to all areas of medicine; however, the great diversity and ever-changing dynamics of perinatal clinical situations - coupled with significant psychological considerations related to caring for patients in such a unique moment of life as the birth of a child - make obstetrics a unique area of medicine. We believe that the solution we should pursue is to create a system of reporting all complications into a single medical database. Only the analysis of such data would allow a reliable assessment of the quality of the procedures currently being performed and give us the grounds for making binding recommendations. It should be noted that not only are the legal regulations - which are a recognised standard in all high-income countries - an essential element here, but most importantly, a "culture of sharing failures" is important - a combination of respect for all patients' rights and, at the same time, respect for the rights and dignity of healthcare professionals. One of the prerequisites for spreading this culture is that medical errors needn't be penalised. Lastly, it should be stressed that serious complications of neuraxial anaesthesia techniques are very rare compared with those in other areas of medicine. Neuraxial anaesthesia is one of the safest medical techniques.

\section{Conflict of interest}

None.

\section{REFERENCES}

1. Wong CA. Advances in labor analgesia. Int J Womens Health. 2010 1: 139-154, doi: 10.2147/ijwh.s4553, indexed in Pubmed: 21072284.

2. Butwick AJ, Bentley J, Wong CA, et al. United States State-Level Variation in the Use of Neuraxial Analgesia During Labor for Pregnant Women. JAMA Netw Open. 2018; 1(8): e186567, doi: 10.1001/jamanetworkopen.2018.6567, indexed in Pubmed: 30646335.

3. D'Angelo R, Smiley RM, Riley ET, et al. Serious complications related to obstetric anesthesia: the serious complication repository project of the Society for Obstetric Anesthesia and Perinatology. Anesthesiology. 2014; 120(6): 1505-1512, doi: 10.1097/ALN.0000000000000253, indexed in Pubmed: 24845921.

4. Ferrari R. Writing narrative style literature reviews. Medical Writing. 2015; 24(4): 230-235, doi: 10.1179/2047480615z.000000000329.

5. von PeltzC, Bennett A, Patil V. Central neurological complications following obstetric neuraxial blockade. Curr Opin Anaesthesiol. 2019; 32(3):315-324, doi: 10.1097/ACO.0000000000000714, indexed in Pubmed: 30789839.

6. Cook TM, Counsell D, Wildsmith JAW, et al. Royal College of Anaesthetists Third National Audit Project. Major complications of central neuraxial block: report on the Third National Audit Project of the Royal College of Anaesthetists. Br J Anaesth. 2009; 102(2): 179-190, doi: 10.1093/bja/aen360, indexed in Pubmed: 19139027.

7. Maronge L, Bogod D. Complications in obstetric anaesthesia. Anaesthesia. 2018; 73 Suppl 1: 61-66, doi: 10.1111/anae.14141, indexed in Pubmed: 29313912

8. Ruppen W, Derry S, McQuay H, et al. Incidence of epidural hematoma, infection, and neurologic injury in obstetric patients with epidural analgesia/anesthesia. Anesthesiology. 2006; 105(2): 394-399, doi: 10.1097/00000542-200608000-00023, indexed in Pubmed: 16871074.

9. Loo CC, Dahlgren G, Irestedt L. Neurological complications in obstetric regional anaesthesia. Int J Obstet Anesth. 2000; 9(2): 99-124, doi: 10.1054/ijoa.1999.0347, indexed in Pubmed: 15321097.

10. Scott DB, Tunstall ME. Serious complications associated with epidural/spinal blockade in obstetrics: a two-year prospective study. Int J Obstet Anesth. 1995; 4(3): 133-139, doi: 10.1016/0959-289x(95)82967-f, indexed in Pubmed: 15636995.

11. Bloom SL, Spong CY, Weiner SJ, et al. National Institute of Child Health and Human Development Maternal-Fetal Medicine Units Network. Complications of anesthesia for cesarean delivery. Obstet Gynecol. 2005; 106(2): 281-287, doi: 10.1097/01.AOG.0000171105.39219.55, indexed in Pubmed: 16055576.

12. Anim-Somuah M, Smyth RMd, Cyna AM, et al. Epidural versus non-epidural or no analgesia for pain management in labour. Cochrane Database Syst Rev. 2018; 5: CD000331, doi: 10.1002/14651858.CD000331.pub4, indexed in Pubmed: 29781504.

13. George RB, Allen TK, Habib AS. Serotonin receptor antagonists for the prevention and treatment of pruritus, nausea, and vomiting in women undergoing cesarean delivery with intrathecal morphine: a systematic review and meta-analysis. Anesth Analg. 2009; 109(1): 174-182, doi: 10.1213/ane.0b013e3181a45a6b, indexed in Pubmed: 19535708.

14. Crowley LJ, Buggy DJ. Shivering and neuraxial anesthesia. Reg Anesth Pain Med. 2008; 33(3): 241-252, doi: 10.1016/j.rapm.2007.11.006, indexed in Pubmed: 18433676. 
15. Goetzl L. Epidural analgesia and maternal fever: a clinical and research update. Curr Opin Anaesthesiol. 2012; 25(3): 292-299, doi: 10.1097/ACO.0b013e3283530d7c, indexed in Pubmed: 22473213.

16. Kinsella SM. A prospective audit of regional anaesthesia failure in 5080 Caesarean sections. Anaesthesia. 2008; 63(8): 822-832, doi: 10.1111/j.1 365-2044.2008.05499.x, indexed in Pubmed: 18547291.

17. Pan PH, Bogard TD, Owen MD. Incidence and characteristics of failures in obstetric neuraxial analgesia and anesthesia: a retrospective analysis of 19,259 deliveries. Int J Obstet Anesth. 2004; 13(4): 227-233, doi: 10.1016/j.ijoa.2004.04.008, indexed in Pubmed: 15477051.

18. Grau T, Leipold RW, Conradi R, et al. Efficacy of ultrasound imaging in obstetric epidural anesthesia. J Clin Anesth. 2002; 14(3): 169-175, doi: 10.1016/s0952-8180(01)00378-6, indexed in Pubmed: 12031746.

19. Headache Classification Committee of the International Headache Society (IHS) The International Classification of Headache Disorders, 3rd edition. Cephalalgia. 2018; 38(1): 1-211, doi: 10.1177/0333102417738202.

20. Goldszmidt E, Kern R, Chaput A, et al. The incidence and etiology of postpartum headaches: a prospective cohort study. Can J Anaesth. 2005; 52(9): 971-977, doi: 10.1007/BF03022061, indexed in Pubmed: 16251565.

21. Choi PT, Galinski SE, Takeuchi L, et al. PDPH is a common complication of neuraxial blockade in parturients: a meta-analysis of obstetrical studies. Can J Anaesth. 2003; 50(5): 460-469, doi: 10.1007/BF03021057, indexed in Pubmed: 12734154.

22. Carvalho B. Respiratory depression after neuraxial opioids in the obstetric setting. Anesth Analg. 2008; 107(3): 956-961, doi: 10.1213/ane.0b013e318168b443, indexed in Pubmed: 18713913.
23. Practice Guidelines for the Prevention, Detection, and Management of Respiratory Depression Associated with Neuraxial Opioid Administration: An Updated Report by the American Society of Anesthesiologists Task Force on Neuraxial Opioids and the American Society of Regional Anesthesia and Pain Medicine. Anesthesiology. 2016; 124(3): 535-552, doi: 10.1097/ALN.0000000000000975, indexed in Pubmed: 26655725.

24. Baer ET. Post-dural puncture bacterial meningitis. Anesthesiology. 2006 105(2): 381-393, doi: 10.1097/00000542-200608000-00022, indexed in Pubmed: 16871073.

25. Davis $L$, Hargreaves $C$, Robinson PN. Postpartum meningitis. Anaesthesia 1993;48(9): 788-789, doi: 10.1111/j.1365-2044.1993.tb07592.x, indexed in Pubmed: 8214499.

26. BeckettVA, Knight M, Sharpe P.The CAPS Study: incidence, management and outcomes of cardiac arrest in pregnancy in the UK: a prospective, descriptive study. BJOG. 2017; 124(9): 1374-1381, doi: 10.1111/14710528.14521, indexed in Pubmed: 28233414.

27. Mhyre JM, Tsen LC, Einav S, et al. Cardiac arrest during hospitalization for delivery in the United States, 1998-2011. Anesthesiology. 2014; 120(4): 810-818, doi: 10.1097/ALN.0000000000000159, indexed in Pubmed: 24694844.

28. Toledo P, Nixon HC, Mhyre JM, et al. Brief report: Availability of lipid emulsion in United States obstetric units. Anesth Analg. 2013; 116(2): 406-408, doi: 10.1213/ANE.0b013e3182713b61, indexed in Pubmed: 23267002. 